\title{
Effects of an extubation readiness test protocol at a tertiary care fully outborn neonatal intensive care unit
}

\author{
Hilal Al Mandhari, ${ }^{1,2}$, Michael Finelli ${ }^{2,3}$, Shiyi Chen ${ }^{4}$, Christopher Tomlinson ${ }^{2}$, Mika L. Nonoyama ${ }^{3,5,6}$
}

\begin{abstract}
H Al Mandhari, M Finelli, S Chen, C Tomlinson, ML Nonoyama. Effects of an extubation readiness test protocol at a tertiary care fully outborn neonatal intensive care unit. Can J Respir Ther 2019;55:81-88. doi: 10.29390/cjrt-2019-011.

Background and objectives: Extubation readiness testing (ERT) in the Neonatal Intensive Care Unit (NICU) is highly variable and lacking standardized criteria. To address this gap, an evidence-based, inter-professionally developed ERT protocol was implemented to assess effectiveness on extubation failure within $72 \mathrm{~h}$ and on duration of intubation (DOI).

Methods: A longitudinal retrospective chart review in a level III, fully outborn NICU, of intubated infants admitted 1-year prior (Group 1), and 1 year after implementation (Group 2). Patients were extubated if they passed a 2-stage ERT protocol (3 min continuous positive airway pressure (CPAP) followed by $7 \mathrm{~min}$ CPAP + pressure support). Descriptive, comparative statistics, and univariate and multiple logistic regression were completed on all patients and a $\leq 326 / 7$ weeks subgroup (intubated at day-of-life 1); $p<0.05$ is considered significant.

Results: All patients $(n=589(n=294$ Group 1, $n=295$ Group 2)) were included (preterm, intubated day of life one subgroup: $n=42$ Group $1, n=38$ Group 2). For all patients, extubation failure decreased significantly from $9.9 \%$ to $4.1 \%(p=0.006)$; Group 1 patients were 2.42 times more likely to experience extubation failure compared with Group 2. Extubation failure in the preterm subgroup decreased from 21.7\% to 2.6\% ( $p=0.01$ ); Group 1 patients were 10.71 times more likely to experience extubation failure. Median DOI was similar in both groups for all patients and in the preterm subgroup.

Conclusions: A unique two-stage ERT protocol was effective at reducing extubation failure rate, without increasing DOI, largely in preterm infants. The evidence-based, interprofessionally developed ERT protocol and its integration into the NICU culture largely contributed to its success.
\end{abstract}

Key Words: extubation readiness; neonates; spontaneous breathing trial

\section{INTRODUCTION}

Intubation is a common procedure in the neonatal intensive care unit (NICU) supporting cardiopulmonary resuscitation, mechanical ventilation, airway protection, meconium aspiration, airway obstruction, medication administration, and perioperative support or management [1]. Removal of an endotracheal tube (ETT) is an important step in a patient's course. If not removed in a timely manner, it can prolong mechanical ventilation past when it is necessary for the infant's wellbeing and can lead to adverse effects such as cardiac and hemodynamic instability, ventilator-induced lung injury, and diaphragmatic muscle weakness [2-6]. Timely removal of the ETT decreases mechanical ventilation duration [5], decreases the need for potentially harmful sedatives [7], and relieves patient and parental distress [8]. On the other hand, extubation failure is a common problem in the NICU, with $20 \%$ to $35 \%$ of preterm and $13 \%$ of term infants failing their first extubation attempt $[9,10]$.

Mechanisms for determining extubation readiness in the NICU are highly variable. Studies have evaluated physiological predictors of extubation failure such as tidal volume and respiratory rate [11, 12], respiratory muscle load and endurance [13, 14], fluid intake and output [15], and electrical diaphragmatic activity [16]. These mechanisms are often associated with other tests such as ETT leak [17, 18], spontaneous breathing trials (SBT) with or without continuous positive airway pressure (CPAP) [5, 19-23], and (or) use of mechanical ventilation technology such as automated (computerized) weaning [24, 25]. This variability is further complicated by the heterogenous definition of extubation failure [26]. The decision to re-intubate may be based on clinical outcomes such as presence of apnea, oxygenation, work of breathing, and (or) arterial blood gases [26]. Additionally, the time period after extubation that is used to define extubation failure varies widely, from 24,48 , or $72 \mathrm{~h}$, and up to 7 or 10 days [27]. In a large international survey on peri-extubation practices (112/162 NICUs), most respondents chose $24(30 \%), 48$ (22\%), or $72 \mathrm{~h}(41 \%)$ [27]. Finally, clinician perceived lack of efficacy or awareness, NICU culture and policies, and overlooking/ overemphasising clinical judgement may contribute to variability in extubation practices [28].

Based on clinical assessment alone, only about $35 \%$ of infants and children ready for extubation are identified [29]. Utilizing specific and objective parameters can decrease extubation failure rates from $16.3 \%$ to $8 \%$ and $9 \%$ [29], but only $16 \%$ of NICUs use a systematic process

\footnotetext{
${ }^{1}$ Neonatal Unit, Child Health department, Sultan Qaboos University Hospital, Muscat, Oman

${ }^{2}$ Neonatology, The Hospital for Sick Children, Toronto, ON, Canada

${ }^{3}$ Department of Respiratory Therapy, The Hospital for Sick Children, Toronto, ON, Canada

${ }^{4}$ Clinical Research Services, The Hospital for Sick Children, Toronto, ON, Canada

${ }^{5}$ Faculty of Health Sciences, University of Ontario Institute of Technology, Oshawa, ON, Canada

${ }^{6}$ Department of Physical Therapy and Rehabilitation Sciences Institute, University of Toronto, Toronto, ON, Canada

Correspondence: Hilal Al Mandhari, Neonatal Unit, Sultan Qaboos University Hospital, P. O. Box:38, Muscat, Al Khoudh P.C 123, Oman. Tel: (+968) 99447092. E-mail: hilalk81@gmail.com
}

Published online at https://www.cjrt.ca on 15 October 2019 
that includes these types of variables [27]. Standardized criteria for extubation readiness testing (ERT) are lacking in the neonatal population due to a dearth of large studies. The European Society for Paediatric and Neonatal Intensive Care gathered an expert panel for a Paediatric mechanical Ventilation Consensus Conference (PEMVECC) [30]. During this conference, 152 recommendations on paediatric and neonatal mechanical ventilation were developed and voted on. PEMVECC reported insufficient to no evidence on all aspects of weaning and extubation processes for critically ill children, basing recommendations on personal experience or adult data [30]. They stated, "At present, there is insufficient paediatric data to provide recommendations on the routine use of any ERT approach that is superior to clinical judgement in invasively ventilated children (strong agreement)" ([30], Supplement p. 15).

Although there is a lack of support for an overarching guide on ERT in the NICU, there is evidence endorsing the use of interprofessionally developed and implemented protocols that consider the social and cultural environment of the unit [28, 31], daily ERT assessments [5], and avoiding pressure support (PS) during SBTs [32, 33]. Evidence has also shown the routine use of an ERT is a strong predictor of extubation success in preterm, low birthweight (BW) infants (sensitivity ranging from $92 \%$ to $97 \%$ ) [19, 34]. Less is known on the success of such testing in full-term infants.

To address the gap in ERT knowledge, an interprofessional team at The Hospital for Sick Children (SickKids), Toronto, Ontario, NICU developed an evidence-based ERT protocol to standardize the process (with objective measures), improve extubation rates, and promote earlier liberation from mechanical ventilation. This study assessed the effectiveness of the ERT protocol in a Canadian tertiary, fully outborn NICU on all infants who were intubated, regardless of gestational age. The primary outcome examined was extubation failure within $72 \mathrm{~h}$, and a secondary outcome duration of intubation (DOI). It was hypothesized that implementation of the ERT protocol would reduce extubation failure by at least $50 \%$, without a change in DOI.

\section{METHODS}

This study was a longitudinal retrospective chart review conducted in the NICU at The Hospital for Sick Children the largest paediatric acute care institution in Canada. It is a level III, 36-bed fully outborn unit with approximately 850 admissions per year (mixed preterm, late preterm, and term infants), and an average of 3500 ventilation days per year. The most common primary diagnoses at admission are surgical anomalies, neurological conditions, and prematurity and related complications.

Prior to the ERT protocol being implemented, infants were fully ventilated on volume-targeted, pressure-control ventilation. Patients were assessed for extubation readiness when they were weaned to minimal ventilation settings and spent varying times on CPAP+PS. Extubation readiness was not consistently discussed at medical rounds, and decisions to extubate were based solely on the clinical judgement of the medical team.

Post-extubation respiratory support for preterm infants $<1250$ grams was nasal CPAP and, if required, nasal noninvasive positive pressure ventilation (NIPPV). For the term infants, a post-extubation, individualized assessment was carried out. If required respiratory support included nasal CPAP, biphasic CPAP, nasal NIPPV, high-flow nasal cannula (HFNC), or on rare occasion, noninvasive high flow oscillatory ventilation (HFOV).

The study was approved by the SickKids Research Ethics Board. A waiver of consent was received because it was a retrospective chart review study.

\section{Patients}

\section{Inclusion criteria}

All intubated infants admitted to the NICU 1 year prior (February 2012 to February 2013, Group 1), and 1 year after (February 2014 to February 2015, Group 2) ERT protocol implementation.

\section{Exclusion criteria}

Infants were excluded if they had unplanned extubation, airway abnormalities, tracheostomy, transferred to another unit with the ETT in situ, or were extubated for palliative care. Patients in Group 2 who had no electronic medical record (EMR) documentation of having had an ERT were excluded. If a patient had more than 1 episode of ventilation, only the first planned extubation was included. The period between Group 1 and 2 (March 2013 to January 2014) was a transition period when clinicians were educated and trained, and the ERT protocol integrated within the culture of the NICU. To avoid bias due to inconsistencies in ERT practices, this transition period was excluded.

\section{Intervention: Extubation readiness test protocol}

The 2-stage ERT Protocol (Figure 1) was developed and supported by an interprofessional team of physicians, respiratory therapists (RRTs) and nurse practitioners. Evidence, clinical expertise, and feasibility (e.g., ease of use) were considered during its development. The original ERT protocol was intended to use ETT CPAP exclusively for $10 \mathrm{~min}$ $[34,35]$ to mimic respiratory muscle load similar to the post-extubation period. However, a large randomized controlled trial [36] using $10 \mathrm{cmH}_{2} \mathrm{O}$ PS (to overcome resistance of the ETT) and clinician preference resulted in the development of a unique two stage ERT protocol: 3 min of CPAP (same positive end expiratory pressure patient was on) followed by $7 \mathrm{~min}$ on PS of $5-8 \mathrm{cmH}_{2} \mathrm{O}$ (to reach spontaneous tidal volume of $4 \mathrm{~mL} / \mathrm{kg}$ ).

After completion, the ERT protocol was combined with the ventilation algorithm of the NICU, incorporated into the EMR and added to the NICU's scorecard as a quality indicator. Extubation and SBT plans were consistently discussed during morning and evening medical rounds. All intubated infants were assessed for SBT eligibility by a RRT twice per day between 8 am and 10 am and $8 \mathrm{pm}$ and $10 \mathrm{pm}$. Absolute contraindications included active necrotizing enterocolitis with decompensation, surgical contraindications, muscle relaxed patients, labile/unstable patients, and some cardiac abnormalities (e.g., single ventricle physiology). Relative contraindications included tension pneumothorax, sepsis, seizure, neuromuscular disorder, recently placed on HFOV or high-frequency jet ventilation and the need for increasing pain management/sedation affecting respiration. There were no notable changes to post-extubation respiratory support strategies over the two time periods.

The ERT protocol was implemented in February 2013. This study aims to examine the impact of introducing this two-stage ERT protocol.

\section{Outcomes}

The main outcome was extubation failure (requiring re-intubation) within $72 \mathrm{~h}$. Other variables included patient characteristics: gestational age (GA), BW, sex, and weight at extubation. Clinical outcomes included fractional inspiratory oxygen $\left(\mathrm{FiO}_{2}\right)$ prior to extubation, DOI, and post-extubation support (CPAP, biphasic CPAP, bilevel NIPPV, low-flow nasal cannula, HFNC, room air, oxyhood).

\section{Data collection}

Data were collected from patients' EMR. Data collected for each patient included BW, GA, sex, underlying diagnosis, age and weight at the time of intubation and extubation, duration of intubation, ventilation parameters before extubation, type of post-extubation support, as well as length of stay (LOS). For the preterm infants, use of antenatal steroids, surfactant, and caffeine as well as presence/absence of intraventricular hemorrhage (IVH), including grade, were collected. The data were collected by a group of 6 volunteer respiratory therapy students.

\section{Statistical analysis}

Descriptive statistics included mean and standard deviation (SD) for continuous variables and median (range) for skewed data. Counts and proportions were calculated for categorical variables (extubation 


\section{FIGURE 1}

SickKids NICU ERT Protocol. CPAP, continuous positive airway pressure; ABG, arterial blood gas; ETT, endotracheal tube; $\mathrm{FiO}_{2}$, fractional inspiratory oxygen; $\mathrm{HR}$, heart rate; $\mathrm{PaCO}_{2}$, partial pressure of arterial carbon dioxide; PEEP. positive end expiratory pressure; PIP, positive inspiratory pressure; PS, pressure support; RR, respiratory rate; SBT, spontaneous breathing trial; VG, volume guarantee; Vt, tidal volume.

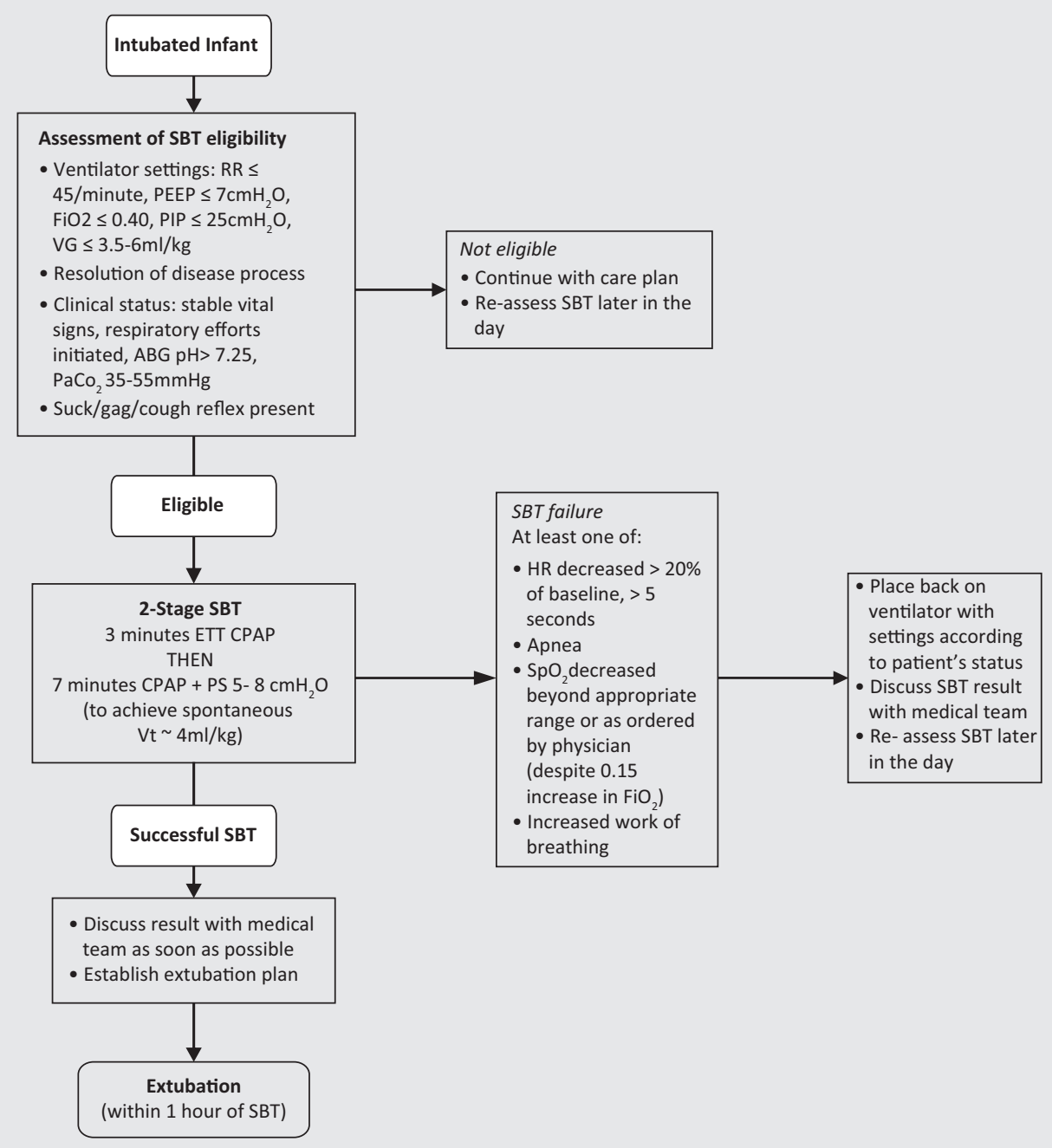

failure, sex, post-extubation support). Statistics comparing patient characteristics and clinical outcomes between Groups (pre- and postERT protocol implementation), were examined using two-sample t-test for normally distributed continuous variables, Wilcoxon two-sample test for non-normally distributed continuous variables, and $\chi^{2}$ test for categorical variables. Univariate and multiple logistic regression analysis were performed to investigate variables associated with extubation failure (odds ratios and $95 \%$ confidence interval (CI)) and DOI (log transformed), adjusted for sex, $\mathrm{FiO}_{2}$ prior to extubation, and GA (model 1) or BW (model 2). Variables used in the models included Group (1 vs. 2), sex (male vs. female), GA (1-week increments), BW (1-kg increments), weight at extubation (1-kg increments), and $\mathrm{FiO}_{2} \times 100$ prior to extubation ( $1 \%$ increments). The same univariate and multiple regression analyses were repeated for a subgroup of preterm infants $\leq 326 / 7$ weeks of gestation who were intubated at day-of-life 1 . This analysis was completed to enhance external validity, as many NICUs and prior studies [19, 34] include only preterm infants.
Statistical analyses were completed using SAS 9.4 (SAS Institute Inc., Cary, NC, USA), and $p<0.05$ was considered significant.

\section{RESULTS}

The total number of eligible patients over the 2 time periods was $n=820$, $n=397$ prior to ERT (Group 1) and $n=423$ after ERT protocol implementation (Group 2). We excluded $n=103$ and $n=128$, leaving $n=294$ and $n=295$ in Group 1 and Group 2, respectively, for the analysis (Figure 2). There were $n=84$ preterm $\leq 326 / 7$ weeks intubated at day-oflife 1, with $n=46$ in Group 1 and $n=38$ in Group 2. The percentage of infants 23-32 6/7 weeks; late preterm, 33-36, 6/7 weeks; and term, $\geq 37$ weeks in Group 1 were, $32.9 \%, 21.4 \%$, and $44.8 \%$, respectively, compared with $28.4 \%, 27.4 \%$ and $44 \%$ in Group 2 . There was no significant difference between the two groups for sex, GA, BW, weight at extubation, $\mathrm{FiO}_{2}$ prior to extubation, and main diagnosis category, for all infants and for preterm infants $\leq 326 / 7$ weeks intubated at day-of-life 1 (Table 1). In addition, there was no difference in the number of preterm infants who received antenatal steroids or had IVH. There was a 


\section{FIGURE 2}

Patient flow diagram. ERT, extubation readiness test; SBT, spontaneous breathing trial.

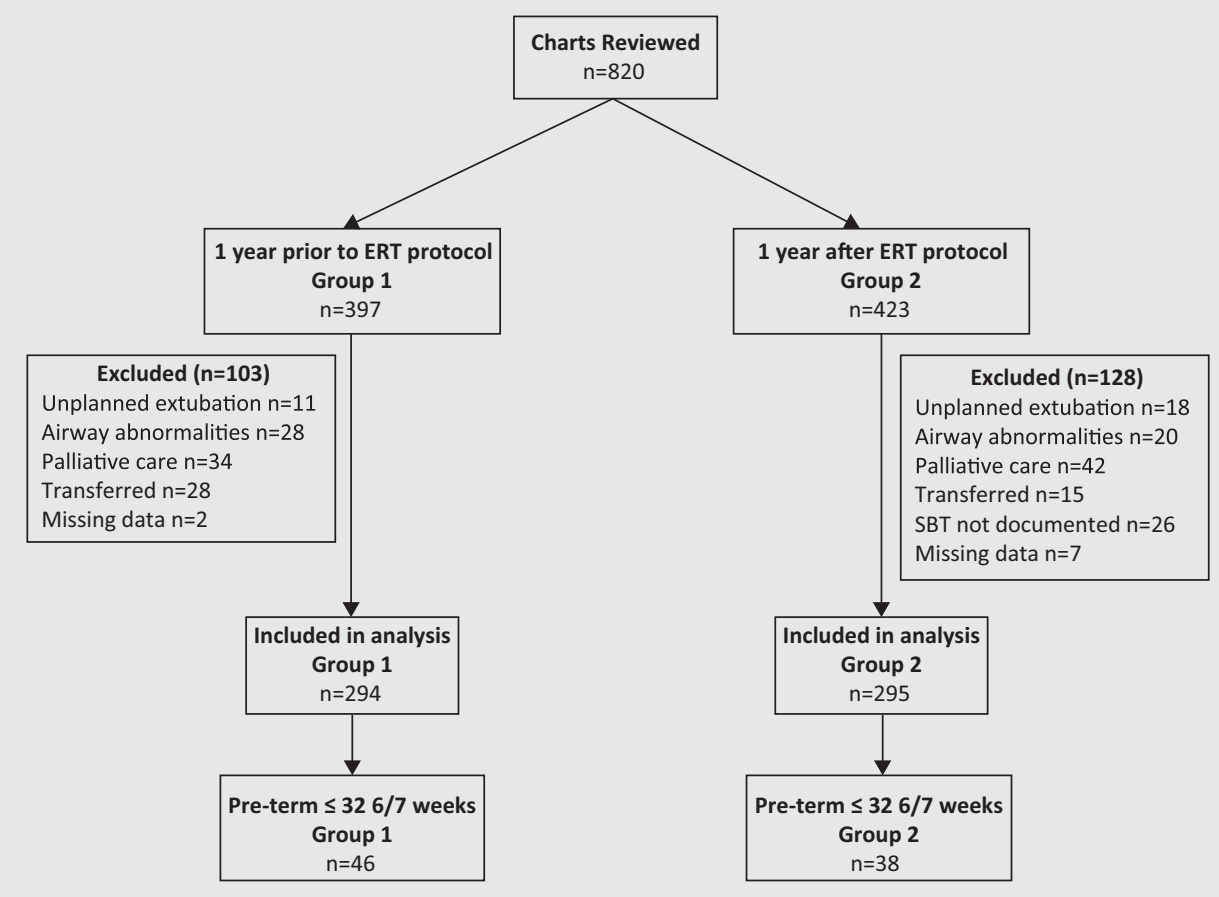

TABLE 1

Characteristics of patients

\begin{tabular}{|c|c|c|c|c|c|c|}
\hline \multirow[b]{2}{*}{ Variable } & \multicolumn{3}{|c|}{ All patients } & \multicolumn{3}{|c|}{ Pre-term $\leq 32$ 6/7 weeks, intubated at day-of-life 1} \\
\hline & Group $1(n=294)$ & Group $2(n=295)$ & $p$ & Group $1(n=46)$ & Group $2(n=38)$ & $p$ \\
\hline Female, $n(\%)$ & $126(42.9)$ & $115(39.3)$ & 0.37 & 20 (43.5) & $13(34.2)$ & 0.39 \\
\hline Birthweight, kg (mean (SD)) & $2.4(1.1)$ & $2.5(0.99)$ & 0.52 & $1.2(0.50$ & $1.2(0.4)$ & 0.72 \\
\hline Weight at extubation, $\mathrm{kg}$ (mean (SD)) & $2.6(1.1)$ & $2.7(0.99)$ & 0.38 & $1.4(0.6)$ & $1.3(0.4)$ & 0.88 \\
\hline $\mathrm{FiO}_{2}$ prior extubation (mean (SD)) & $0.23(0.1)$ & $0.23(0.1)$ & 0.14 & $0.27(0.1)$ & $0.23(0.1)$ & 0.17 \\
\hline Received antenatal steroids, $n(\%)$ & - & - & - & $21(46.7)$ & $21(56.8)$ & 0.36 \\
\hline \multicolumn{7}{|l|}{ Post-extubation support ${ }^{\mathrm{b}}$} \\
\hline Room air & $160(54.4)$ & $170(57.6)$ & - & $6(13.0)$ & $10(26.3)$ & - \\
\hline CPAP & $59(20.1)$ & $79(26.8)$ & - & $24(52.2)$ & $18(47.4)$ & - \\
\hline Biphasic CPAP & $5(2.5)$ & 0 & - & $2(4.4)$ & 0 & - \\
\hline LFNC & $40(13.6)$ & $14(4.8)$ & - & $1(2.2)$ & $1(2.6)$ & - \\
\hline HFNC & $6(2.0)$ & $2(0.7)$ & - & 0 & 0 & - \\
\hline NIPPV & $20(6.8)$ & $28(9.5)$ & - & $10(21.7)$ & $8(21.1)$ & - \\
\hline Infectious & $9(3)$ & $6(2)$ & - & - & - & - \\
\hline Cardiac & $7(3.1)$ & $5(1.7)$ & - & - & - & - \\
\hline Neurologic & $41(13.9)$ & $58(19.7)$ & - & - & - & - \\
\hline Genetic/metabolic & $10(3.4)$ & $12(4.1)$ & - & - & - & - \\
\hline Surgical & $61(20.7)$ & $64(21.7)$ & - & - & - & - \\
\hline Prematurity and related complications & $118(40.1)$ & $106(35.9)$ & - & - & - & - \\
\hline Length of stay, days (Median (range)) & $13(1-199)$ & $11(2-221)$ & 0.29 & $32(3-199)$ & $23(3-123)$ & 0.30 \\
\hline
\end{tabular}

${ }^{a} \mathrm{n}=2$ missing from Group 1.

${ }^{b}$ unable to run Chi-Square test because of low cell count.

Note: CPAP, continuous positive airway pressure; $\mathrm{FiO}_{2}$, fractional inspiratory oxygen; HFNC, high-flow nasal cannula; IVH, intraventicular hemorrhage; LFNC,

low-flow nasal cannula; NI-HFOV, noninvasive high-flow oscillatory ventilation; NIPPV, noninvasive positive pressure ventilation. 
significant difference in the number of preterm infants who received surfactant $n=38(82.6 \%)$ in Group 1 compared with $n=24(63.2 \%)$ in Group 2, $p=0.04$ (Table 1).

For all patients, extubation failure decreased significantly from 29/294 (9.9\%) in Group 1 to 12/295 (4.1\%) in Group 2, $p=0.006$. Similar results were found for preterm infants intubated at day-of-life 1 , with extubation failure significantly decreasing from 10/46 (21.7\%) in Group 1 to $1 / 38$ (2.6\%) in Group 2, $p=0.01$ (Table 2). The median DOI was similar in both groups for all patients ( 3 days, $p=0.55)$ and for preterm infants intubated at day-of-life 1 ( 2 days in Group 1 and 1 day in Group 2, $p=0.18$ ) (Table 2). The median LOS was also similar in both groups for all infants (13 days in Group 1 compared with 11 days in Group 2, $p=0.29$ ). Similarly, there was no significant difference in median LOS for preterm infants intubated day-of-life 1 (32 days for group 1 vs. 23 days for group 2, $p=0.30)$.

When all intubated infants (regardless of intubated day) were stratified by GA, there was a significant decrease in extubation failure between Groups for preterm infants $\leq 326 / 7$ weeks: 16/97 (16.5\%) in Group 1 to $5 / 84(6.0 \%)$ in Group 2, $p=0.02$. Though there was no statistical significance, there was a reduction between the 2 groups for older infants GA 233 weeks: $13 / 195(6.7 \%)$ in Group 1 to $7 / 211$ (3.3\%) in Group 2, $p=0.12$.

Multiple regression results for extubation failure for all patients are found in Table 3. Weight at extubation was highly correlated with GA and $\mathrm{BW}$ in the univariate analysis and excluded from the multiple regression models. All patients in Group 1 were 2.42 times more likely to experience extubation failure compared with Group 2, $p=0.02$. Similar results were found with model 2 that included BW instead of GA: Group 1 was 2.69 times more likely to experience extubation failure compared with Group 2, $p=0.008$. For model 1, as GA increased by 1 week, the odds of extubation failure decreased by $11 \%, p=0.0006$ and as the $\mathrm{FiO}_{2} \times 100$ prior to extubation increased by $1 \%$, extubation failure increased by $5 \%, p=0.02$. For model 2 as BW increased by $1 \mathrm{~kg}$, the odds of extubation failure decreased by $35 \%, p=0.0092$, and as the $\mathrm{FiO}_{2}$ $\times 100$ prior to extubation increased by $1 \%$, extubation failure increased by $5 \%, p=0.03$. Sex was not associated with extubation failure in either model.

Multiple logistic regression results for extubation failure, in preterm $\leq 326 / 7$ weeks intubated on day-of-life 1 , are found in Table 4. Only 2 variables (Group and GA) were included in the multiple regression models due to smaller sample size $(n=84)$ and event rate $(n=11)$. Although surfactant use was significantly lower in Group 2, on univariate logistic regression analysis this difference was not significant (OR 1.70, CI 0.34-8.55, $p=0.52$ ). Hence, surfactant use was not included in the multiple logistic regression analysis model. All patients in Group 1 were 10.71 times more likely to experience extubation failure compared with Group 2, $p=0.04$. Similar results were found with model 2: Group 1 was 13.83 times more likely to experience extubation failure compared with Group 2, $p=0.02$. For model 1 , as GA increased by 1 week, the odds of extubation failure decreased by $47 \%, p=0.002$. For model 2 as BW increased by $1 \mathrm{~kg}$, the odds of extubation failure decreased by $99.6 \%$, $p=0.004$.

Multiple regression results for DOI in all patients are found in Table 5. Weight at extubation was highly correlated with GA and BW in the univariate analysis and was excluded from the multiple regression models. For model 1 , as GA increased by 1 week, the DOI decreased by $4 \%$ $(p<0.0001)$ and as $\mathrm{FiO}_{2} \times 100$ prior extubation increased by $1 \%$, the DOI increased by $7 \%(p<0.0001)$. Similar results were for found for model 2: as BW increased by $1 \mathrm{~kg}$, DOI decreased by $14 \%(p<0.0001)$ and as $\mathrm{FiO}_{2} \times 100$ prior extubation increased by $1 \%$, the DOI increased by $7 \%(p<0.0001)$. Group and sex were not associated with DOI in either model.

Multiple regression results for DOI, in preterm $\leq 32$ 6/7 weeks intubated day-of-life 1 , are found in Table 6 . For model 1, as GA increased by 1 week, the DOI decreased by $18 \%(p=0.0002)$ and as $\mathrm{FiO}_{2} \times 100$ prior extubation increased by $1 \%$, the DOI increased by $6 \%(p<0.0001)$. Similar results were found for model 2: as BW increased by $1 \mathrm{~kg}$, DOI decreased by $56 \%(p=0.01)$ and as $\mathrm{FiO}_{2} \times 100$ prior extubation increased by $1 \%$, the DOI increased by $7 \%(p<0.0001)$. Group and sex were not associated with DOI in either model.

TABLE 2

Extubation failure and duration of intubation

\begin{tabular}{|c|c|c|c|c|c|c|}
\hline \multirow[b]{2}{*}{ Variable } & \multicolumn{3}{|c|}{ All patients } & \multicolumn{3}{|c|}{ Pre-term $\leq 326 / 7$ weeks intubated day of life one } \\
\hline & Group $1(n=294)$ & Group $2(n=295)$ & $p$ & Group $1(n=46)$ & Group $2(n=38)$ & $p$ \\
\hline Extubation failure, $n(\%)$ & $29(9.9)$ & $12(4.1)$ & 0.006 & $10(21.7)$ & $1(2.6)$ & 0.01 \\
\hline $\begin{array}{l}\text { Duration of intubation, days } \\
\text { (median (range)) }\end{array}$ & $3(0.25-74)$ & $3(0.24-80)$ & 0.55 & $2(0.4-80)$ & $1(0.5-80)$ & 0.18 \\
\hline
\end{tabular}

TABLE 3

Multiple regression all patients, extubation failure

\begin{tabular}{lccc}
\hline & Odds ratio & $\mathbf{9 5 \%} \mathbf{C l}$ & $\boldsymbol{p}$ \\
\hline Model 1 (41/573 events) & & & \\
Group (1 vs. 2) & 2.42 & $1.19-4.94$ & 0.02 \\
Gestational age (1 week) & 0.89 & $0.84-0.95$ & 0.0006 \\
Sex (male vs. female) & 0.76 & $0.38-1.53$ & 0.45 \\
$\mathrm{FiO}_{2}$ prior to extubation $\times 100$ (1 unit) & 1.05 & $1.01-1.10$ & 0.02 \\
Model 2 (40/575 events) & & & \\
Group (1 vs. 2) & 2.69 & $1.30-5.56$ & 0.008 \\
Birth weight (1 kg) & 0.65 & $0.47-0.90$ & 0.009 \\
Sex (male vs. female) & 0.66 & $0.33-1.33$ & 0.25 \\
$\mathrm{FiO}_{2}$ prior to extubation $\times 100$ (1 unit) & 1.05 & $1.01-1.10$ & 0.03 \\
\hline
\end{tabular}

Note: Adjusted for sex, fractional inspiratory oxygen $\left(\mathrm{FiO}_{2}\right)$ prior to extubation, and gestational age (model 1) or birth weight (model 2).

\section{TABLE 4}

Multiple Regression for preterm $\leq 32$ 6/7 weeks intubated day-of-life 1 , extubation failure

\begin{tabular}{lccc}
\hline & Odds ratio & $95 \% \mathrm{Cl}$ & $\boldsymbol{p}$ \\
\hline Model 1 (11/84 events) & & & \\
Group (1 vs. 2) & 10.71 & $1.13-101.23$ & 0.04 \\
Gestational age (1 week) & 0.53 & $0.35-0.80$ & 0.002 \\
Model 2 (11/84 events) & & & \\
Group (1 vs. 2) & 13.83 & $1.41-135.91$ & 0.02 \\
Birth weight (1 kg) & 0.004 & $<0.001-0.18$ & 0.004 \\
\hline
\end{tabular}

Note: Adjusted for sex, fractional inspiratory oxygen prior to extubation, and gestational age (model 1) or birth weight (model 2). 


\section{TABLE 5}

Multiple regression all patients, duration of intubation (days)

\begin{tabular}{lrlrc}
\hline & Estimate & Standard error & $\boldsymbol{t}$ & $\boldsymbol{p}$ \\
\hline Model 1 (41/573 events) & & & & \\
Group (1 vs. 2) & 0.03 & 0.08 & 0.33 & 0.74 \\
Gestational age (1 week) & -0.04 & 0.009 & -4.74 & $<0.0001$ \\
Sex (male vs. female) & -0.02 & 0.08 & -0.27 & 0.79 \\
$\mathrm{FiO}_{2}$ prior to extubation & 0.07 & 0.008 & 8.62 & $<0.0001$ \\
$\times 100$ (1 unit) & & & & \\
Model 2 (40/575 events) & & & & \\
Group (1 vs. 2) & 0.01 & 0.08 & 0.10 & 0.92 \\
Birth weight (1 kg) & -0.15 & 0.04 & -3.93 & $<0.0001$ \\
Sex (male vs. female) & -0.03 & 0.08 & -0.35 & 0.72 \\
$\mathrm{FiO}_{2}$ prior to extubation $\times$ & 0.07 & 0.008 & 9.66 & $<0.0001$ \\
100 (1 unit) & & & & \\
\hline
\end{tabular}

Note: Log transformed, adjusted for sex, fractional inspiratory oxygen $\left(\mathrm{FiO}_{2}\right)$ prior to extubation, and gestational age (model 1) or birth weight (model 2).

\section{TABLE 6}

Multiple Regression for preterm $\leq 326 / 7$ weeks intubated day-of-life 1, duration of intubation (days)

\begin{tabular}{lrrrc}
\hline & Estimate & Standard error & $\boldsymbol{t}$ & $\boldsymbol{p}$ \\
\hline Model 1 ( $\boldsymbol{n}=\mathbf{8 2}$ ) & & & & \\
Group (1 vs. 2) & 0.13 & 0.25 & 0.54 & 0.59 \\
Gestational age (1 week) & -0.20 & 0.05 & -3.92 & 0.0002 \\
Sex (male vs. female) & -0.48 & 0.25 & -1.92 & 0.06 \\
$\mathrm{FiO}_{2}$ prior to extubation & 0.06 & 0.01 & 5.15 & $<0.0001$ \\
100 (1 unit) & & & & \\
Model 2 ( $\boldsymbol{n}=\mathbf{8 2})$ & & & & \\
Group (1 vs. 2) & 0.20 & 0.26 & 0.75 & 0.45 \\
Birth weight (1 kg) & -0.81 & 0.32 & -2.54 & 0.01 \\
Sex (male vs. female) & -0.53 & 0.26 & -2.02 & 0.05 \\
FiO prior to extubation $\times$ & 0.07 & 0.01 & 5.32 & $<0.0001$ \\
100 (1 unit) & & & &
\end{tabular}

Note: Log transformed, adjusted for sex, fractional inspiratory oxygen $\left(\mathrm{FiO}_{2}\right)$ prior to extubation, and gestational age (model 1) or birth weight (model 2).

\section{DISCUSSION}

This study showed an evidence-based, interprofessionally developed NICU ERT protocol was effective at reducing extubation failure rate from $9.9 \%$ to $4.1 \%$. Infants were about 2.5 times more likely to have extubation failure during the period when no ERT protocol existed, compared with when one existed (accounting for GA, BW and $\mathrm{FiO}_{2}$ prior to extubation). Our study revealed much lower rates of extubation failure ( $4.1 \%$ in all patients, $2.6 \%$ in preterm infants), but our similar magnitudes of reduction (about 50\%), compared with other studies [10, 34]. Hermeto et al. [10] found a reduction in extubation failure from $40 \%$ (prior to SBT implementation) to 26\% (1 year) and 20\% (2 years) post-implementation. Andrade et al. [20] showed a difference of $33.3 \%$ with an SBT protocol compared with $63.3 \%$ without an SBT protocol. Although extubation failure was reduced, the DOI remained the same (median 3 days). Early extubation may be favoured, but this DOI was likely the optimal period to ensure extubation success, avoid re-intubation, and repeated courses of mechanical ventilation [10, 35].

Infants who were pre-term $\leq 326 / 7$ weeks and intubated on day-oflife 1 drove the results of this study. The reduction in extubation failure decreased from $21.7 \%$ to $2.6 \%$, and they were $11-14$ times more likely to have extubation failure during the time when no ERT protocol existed, compared with when one existed. For older infants with GA greater than 33 weeks there was also a reduction in extubation failure, but this result was not statistically significant (6.7\% in Group 1 to $3.3 \%$ in Group 2).
Most research of ERT protocols are in younger, smaller infants [10, 19, $20,34,35]$ or older children $[11,36,37]$ and we could not find studies describing ERT protocols in older infants. Future research in extubation and weaning practices should target this subgroup of neonates.

The unique 10-min two-stage SBT used in this study was not only guided by evidence $[21,35,36]$ but also equally by clinical expertise. Several different iterations of ERT protocols [10-12, 19, 20, 34-37] have been investigated in the past, each showing success at reducing extubation failure rates and (or) duration of mechanical ventilation. It is likely that both our ERT protocol and the consistent and systematic use is what contributed to its success $[28,38]$. The ERT protocol became a part of the SickKids NICU common vocabulary and routine, was adopted readily by the medical team, and allowed for changes over time [39]. This included discussions of factors associated with extubation failure such as GA, BW, and level of ventilator support. In this study there were decreased odds of extubation failure and reduced DOI in more mature and heavier infants (regardless of GA). There was also increased odds of extubation failure and increased DOI if the $\mathrm{FiO}_{2}$ prior to extubation was higher. These results have been found in other studies assessing predictors of extubation failure $[9,40,41]$.

The strength of this study was the large sample size of patients $(n=589)$ within a fully outborn NICU that included a mix of both term and preterm infants. However, the study also has a number of limitations that should be addressed in future trials. One limitation is the biases associated with a retrospective study design [42]. The extubation failure events were low in number, especially in the preterm subgroup, and small changes might have significantly changed the statistical inferences. Although there were no notable changes in post-extubation support over the study period, changes in clinician practices, and technology advances (NIPPV, HFNC) may have contributed to the results. Indeed, we found surfactant use decreased significantly over the 2 time periods. Post-extubation interventions may have been a confounding factor in our study. Though post-extubation interventions were collected, influence on post-extubation success or failure was not assessed due to multiple low cell counts. However, on univariate regression analysis the difference in surfactant use was not statistically significant $(p=0.52)$. Consequently, surfactant use was not included in the multiple logistic regression analysis models because the difference is not significant on univariate logistic regression as well as smaller sample size $(n=84)$ and event rate $(n=11)$. We did not include other variables potentially associated with extubation failure such as the indication for intubation, severity of disease, ventilation and physiological parameters [9, 14, 43, 44], and clinician behaviour [28]. Long-term outcomes such as bronchopulmonary dysplasia, mortality, economic benefits, and infant and family considerations (e.g., comfort) were not described. Data on infants who were not eligible for or failed the SBT were not collected, though comparisons between time periods would not be possible since an SBT did not exist before the ERT protocol was put in place. However, Group 2 patients who did not have ERT documented in their chart were excluded. Physiological variables and adverse effects during the ERT were not collected. Although the ERT protocol was well integrated into the NICU culture, compliance was not assessed and some extubations may have occurred outside the ERT algorithm. Generalizability may be limited because only the SickKids NICU culture and policies were considered during ERT protocol development. In addition, the population was more mature, with lower prevalence of lung disease as evidenced by the low event rate and the outborn nature of the unit.

\section{CONCLUSION}

A unique two-stage 10-min ERT protocol in a tertiary care fully outborn NICU was effective at reducing extubation failure rate, without increasing duration of intubation. The effect was largely seen in preterm infants, and future research is encouraged to confirm the reduced rate of extubation failure found in term infants cared for in the NICU. The evidence-based, interprofessionally developed ERT protocol and its implementation and integration into the NICU culture largely contributed to its success. 


\section{ACKNOWLEDGEMENTS}

We acknowledge the contribution of 6 students from the Michener Institute for Applied Health Sciences Respiratory Therapy program for the data collection: Tina Yan, Sagel Osman, Richa Shah, Shirley Quach, Kajal Patel, and Mirjana Tusha.

\section{CONTRIBUTORS}

HM contributed to initial concept of the study, HM, MF, CT contributed to the study design, The data were collected by volunteer RT students with help and support from HM and MF. SC, HM, MF, and CT contributed to analysis, and interpretation of the data. HM, MF, CT, $\mathrm{MN}$ contributed to initial draft, review and final approval of manuscript.

\section{FUNDING}

This study did not receive any specific grant from funding agencies in the public, commercial, or not-for-profit sectors.

\section{COMPETING INTERESTS}

All authors have completed the ICMJE uniform disclosure form at www. icmje.org/coi_disclosure.pdf and declare no financial relationships with any organizations that might have an interest in the submitted work in the previous 3 years; no other relationships or activities that could appear to have influenced the submitted work.

\section{REFERENCES}

1. Maheshwari R, Tracy M, Badawi N, Hinder M. Neonatal endotracheal intubation: How to make it more baby friendly. J Paediatr Child Health 2016;52(5): 480-6. doi: 10.1111/jpc.13192.

2. Centre for Disease Control and Prevention. Ventilator-Associated Event (VAE) Protocol 2016. Available at: http://www.cdc.gov/nhsn/PDFs/pscManual/10-VAE_FINAL.pdf (Accessed January 2019).

3. Foglia E, Meier MD, Elward A. Ventilator-associated pneumonia in neonatal and pediatric intensive care unit patients. Clin Microbiol Rev 2007;20(3): 409-25table of contents.. doi: 10.1128/CMR.00041-06.

4. Klompas M, Li L, Kleinman K, Szumita PM, Massaro AF. Associations between ventilator bundle components and outcomes. JAMA Intern Med 2016;176(9): 1277-83. doi: 10.1001/jamainternmed.2016.2427.

5. Newth CJ, Venkataraman S, Willson DF, et al. Weaning and extubation readiness in pediatric patients. Pediatr Crit Care Med 2009;10(1): 1-11. doi: 10.1097/PCC.0b013e318193724d.

6. Valenzuela J, Araneda P, Cruces P. Weaning from mechanical ventilation in paediatrics. State of the art. Arch Bronconeumol 2014;50(3): 105-12. doi: 10.1016/j.arbres.2013.02.003.

7. Curley MA, Wypij D, Watson RS, et al. Protocolized sedation vs usual care in pediatric patients mechanically ventilated for acute respiratory failure: A randomized clinical trial. JAMA 2015;313(4): 379-89. doi: 10.1001/jama.2014.18399.

8. Haines C, Perger C, Nagy S. A comparison of the stressors experienced by parents of intubated and non-intubated children. J Adv Nurs 1995;21(2): 350-5. doi: 10.1111/j.1365-2648.1995.tb02533.x.

9. Costa AC, Schettino Rde C, Ferreira SC. [Predictors of extubation failure and reintubation in newborn infants subjected to mechanical ventilation]. Rev Bras Ter Intensiva 2014;26(1): 51-6. doi: 10.5935/ 0103-507X.20140008.

10. Hermeto F, Bottino MN, Vaillancourt K, Sant'Anna GM. Implementation of a respiratory therapist-driven protocol for neonatal ventilation: Impact on the premature population. Pediatrics 2009;123(5): e907-16. doi: 10.1542/peds.2008-1647.

11. Farias JA, Alia I, Esteban A, Golubicki AN. Olazarri FA. Weaning from mechanical ventilation in pediatric intensive care patients. Intensive Care Med 1998;24(10): 1070-5. doi: 10.1007/s001340050718.

12. Gillespie LM, White SD, Sinha SK, Donn SM. Usefulness of the minute ventilation test in predicting successful extubation in newborn infants: A randomized controlled trial. J Perinatol 2003;23(3): 205-7. doi: 10.1038/ sj.jp.7210886.

13. Noizet O, Leclerc F, Sadik A, et al. Does taking endurance into account improve the prediction of weaning outcome in mechanically ventilated children? Crit Care (London, England 2005;9(6): R798-807. doi: $10.1186 / \mathrm{cc} 3898$.

14. Harikumar G, Egberongbe Y, Nadel S, et al. Tension-time index as a predictor of extubation outcome in ventilated children.
Am J Respir Crit Care Med 2009;180(10): 982-8. doi: 10.1164/ rccm.200811-1725OC.

15. Randolph AG, Forbes PW, Gedeit RG, et al. Cumulative fluid intake minus output is not associated with ventilator weaning duration or extubation outcomes in children. Pediatr Crit Care Med 2005;6(6): 642-7. doi: 10.1097/01.PCC.0000185484.14423.0D.

16. Wolf GK, Walsh BK, Green ML, Arnold JH. Electrical activity of the diaphragm during extubation readiness testing in critically ill children. Pediatr Crit Care Med 2011;12(6): e220-4. doi: 10.1097/ PCC.0b013e3181fe28fc.

17. Wratney AT, Benjamin DK, Jr., Slonim AD, He J, Hamel DS, Cheifetz IM. The endotracheal tube air leak test does not predict extubation outcome in critically ill pediatric patients. Pediatr Crit Care Med 2008;9(5): 490-6. doi: 10.1097/PCC.0b013e3181849901.

18. Suominen PK, Tuominen NA, Salminen JT, et al. The air-leak test is not a good predictor of postextubation adverse events in children undergoing cardiac surgery. J Cardiothorac Vasc Anesth 2007;21(2): 197-202. doi: $10.1053 /$ j.jvca.2006.01.007.

19. Chawla S, Natarajan G, Gelmini M, Kazzi SN. Role of spontaneous breathing trial in predicting successful extubation in premature infants. Pediatr Pulmonol 2013;48(5): 443-8. doi: 10.1002/ppul.22623.

20. Andrade LB, Melo TM, Morais DF, Lima MR, Albuquerque EC, Martimiano PH. Spontaneous breathing trial evaluation in preterm newborns extubation. Rev Bras Ter Intensiva 2010;22(2): 159-65. doi: 10.1590/S0103-507X2010000200010.

21. Davis PG, Henderson-Smart DJ. Extubation from low-rate intermittent positive airways pressure versus extubation after a trial of endotracheal continuous positive airways pressure in intubated preterm infants. Cochrane Database Syst Rev 2001;(4): Cd001078. doi: 10.1002/14651858. CD001078.

22. Boles JM, Bion J, Connors A, et al. Weaning from mechanical ventilation. Eur Respir J 2007;29(5): 1033-56. doi: 10.1183/09031936.00010206.

23. Robertson TE, Mann HJ, Hyzy R, et al. Multicenter implementation of a consensus-developed, evidence-based, spontaneous breathing trial protocol. Crit Care Med 2008;36(10): 2753-62. doi: 10.1097/ CCM.0b013e3181872833.

24. Jouvet P, Eddington A, Payen V, et al. A pilot prospective study on closed loop controlled ventilation and oxygenation in ventilated children during the weaning phase. Crit Care (London, England) 2012;16(3): R85. doi: 10.1186/cc11343.

25. Rose L, Schultz MJ, Cardwell CR, Jouvet P, McAuley DF, Blackwood B. Automated versus non-automated weaning for reducing the duration of mechanical ventilation for critically ill adults and children: A cochrane systematic review and meta-analysis. Crit Care (London, England) 2015;19: 48. doi: 10.1186/s13054-015-0755-6.

26. Shalish W, Sant' Anna GM, Natarajan G, Chawla S. When and how to extubate premature infants from mechanical ventilation. Curr Pediatr Rep 2014;2(1): 18-25. doi: 10.1007/s40124-013-0032-6.

27. Al-Mandari H, Shalish W, Dempsey E, Keszler M, Davis PG, Sant'anna G. International survey on periextubation practices in extremely preterm infants. Archiv Dis Child Fetal Neonatal Ed 2015;100(5): F428-31. doi: 10.1136/archdischild-2015-308549.

28. Jordan J, Rose L, Dainty KN, Noyes J, Blackwood B. Factors that impact on the use of mechanical ventilation weaning protocols in critically ill adults and children: A qualitative evidence-synthesis. Cochrane Database Syst Rev 2016;Oct 4;10:CD011812. doi: 10.1002/14651858.CD011812.pub2.

29. Bankhead S, Kolea C, Kamai S. Preventing extubation failures in a pediatric intensive care unit. Nurs Clin North Am 2014;49(3): 321-8. doi: 10.1016/j.cnur.2014.05.006.

30. Kneyber MCJ, de Luca D, Calderini E, et al. Recommendations for mechanical ventilation of critically ill children from the Paediatric Mechanical Ventilation Consensus Conference (PEMVECC). Intensive Care Med 2017:43: 1764-1780.

31. Blackwood B, Murray M, Chisakuta A, Cardwell CR, O'Halloran P. Protocolized versus non-protocolized weaning for reducing the duration of invasive mechanical ventilation in critically ill paediatric patients. Cochrane Database Syst Rev 2013;7): Art. No.: CD009082. doi: 10.1002/14651858.CD009082.pub2.

32. Manczur T, Greenough A, Nicholson GP, Rafferty GF. Resistance of pediatric and neonatal endotracheal tubes: Influence of flow rate, size, and shape. Crit Care Med 2000;28(5): 1595-8. doi: 10.1097/00003246-200005000-00056.

33. Tobin MJ. Extubation and the myth of "minimal ventilator settings". Am J Respir Crit Care Med 2012;185(4): 349-50. doi: 10.1164/ rccm.201201-0050ED. 
34. Kamlin CO, Davis PG, Morley CJ. Predicting successful extubation of very low birthweight infants. Arch Dis Child Fetal Neonatal Ed 2006;91(3): F180-3. doi: 10.1136/adc.2005.081083.

35. Kamlin COF, Davis PG, Argus B, Mills B, Morley CJ. A trial of spontaneous breathing to determine the readiness for extubation in very low birth weight infants: A prospective evaluation. Arch Dis Child Fetal Neonatal Ed 2008;93(4): F305-6. doi: 10.1136/adc.2007.129890.

36. Foronda FK, Troster EJ, Farias JA, et al. The impact of daily evaluation and spontaneous breathing test on the duration of pediatric mechanical ventilation: A randomized controlled trial. Crit Care Med 2011;39(11): 2526-33. doi: 10.1097/CCM.0b013e3182257520.

37. Chavez A, dela Cruz R, Zaritsky A. Spontaneous breathing trial predicts successful extubation in infants and children. Pediatr Crit Care Med 2006;7(4): 324-8. doi: 10.1097/01.PCC.0000225001.92994.29.

38. Solberg MT, Hansen TW, Bjork IT. The need for predictability in coordination of ventilator treatment of newborn infants - a qualitative study. Intensive Crit Care Nurs 2015;31(4): 205-12. doi: 10.1016/j. iccn.2014.12.003.

39. Ely EW, Meade MO, Haponik EF, et al. Mechanical ventilator weaning protocols driven by nonphysician health-care professionals: Evidence-based clinical practice guidelines. Chest 2001;120: (6 Suppl): 454s-63s. doi: 10.1378/chest.120.6_suppl.454S.

40. Laham JL, Breheny PJ, Rush A. Do clinical parameters predict first planned extubation outcome in the pediatric intensive care unit? J Intensive Care Med 2015;30(2): 89-96. doi: 10.1177/0885066613494338.

41. Venkataraman ST, Khan N, Brown A. Validation of predictors of extubation success and failure in mechanically ventilated infants and children. Crit Care Med 2000;28(8): 2991-6. doi: 10.1097/00003246-200008000-00051.

42. Cummings S, Kohn M, Hulley S. Designing cross-sectional and cohort studies. designing clinical research. 4th edn. Lippincott Williams \& Wilkins, Philadelphia, PA; 2013. p. 85-96.

43. Kaczmarek J, Chawla S, Marchica C, Dwaihy M, Grundy L, Sant'Anna GM. Heart rate variability and extubation readiness in extremely preterm infants. Neonatology 2013;104(1): 42-8. doi: 10.1159/000347101.

44. Szymankiewicz M, Vidyasagar D, Gadzinowski J. Predictors of successful extubation of preterm low-birth-weight infants with respiratory distress syndrome. Pediatr Crit Care Med 2005;6(1): 44-9. doi: 10.1097/01. PCC.0000149136.28598.14 